\title{
Quantifying Sources and Types of Smartwatch Usage Sessions
}

\author{
Anonymised
}

\begin{abstract}
We seek to quantify smartwatch use, and establish differences and similarities to smartphone use. Our analysis considers use traces from 307 users that include over 2.8 million notifications and 800,000 screen usage events, and we compare our findings to previous work that quantifies smartphone use. The results show that smartwatches are used more briefly and more frequently throughout the day, with half the sessions lasting less than 5 seconds. Interaction with notifications is similar across both types of devices, both in terms of response times and preferred application types. We also analyse the differences between our smartwatch dataset and a dataset aggregated from four previously conducted smartphone studies. The similarities and differences between smartwatch and smartphone use suggest effect on usage that go beyond differences in form factor.
\end{abstract}

\section{Author Keywords}

Smartwatches; smartphones; usage; session; interactions; applications.

\section{ACM Classification Keywords}

H.5.m. Information interfaces and presentation (e.g., HCI): Miscellaneous.

\section{INTRODUCTION}

This large-scale longitudinal study focuses on quantifying how smartwatches are used in daily life. This work aims to understand whether smartwatches are actually used as devices in their own right, or as a mere extension to smartphones. Current smartwatches enable users to synchronise notifications, interact with their smartwatch applications, and display short pieces of information. Understanding the similarities and differences in smartwatch and smartphone use can inform us about how this technology is appropriated by end-users, and whether current interaction methods are suitable. Should we design smartwatches as independent devices, or as smartphone extensions?

While smartwatches have existed for nearly two decades in various forms, they have yet to be widely adopted by the

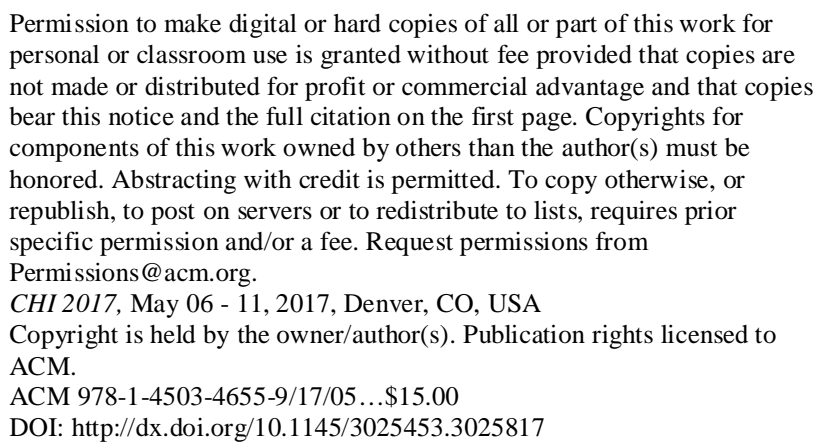

general population. This may be due to lack of a "killer app" sufficiently distinguishing the smartwatch from the smartphone [16], the fact that long-term added benefits of using smartwatches are still unclear, or the (technical) limitations of smartwatches (i.e., network and battery limitations, smaller screen size). As a result, use cases for smartwatches are still scarce, and consumer benefits and acceptance have not been extensively studied. Smartwatches need to combine two requirements [16]: provide digital information and functionality of a traditional watch, as well as the ability for personal expression. Wu et al. [39] surveyed smartwatch users and concluded that the largest impact users desire from smartwatches are visible and tangible benefits when used.

Further, Min et al. [20] report on differences in desired smartwatch functionality between novel users (less than 3 months of smartwatch usage) vs. experienced users, with novel users prioritising time keeping, while experienced users prioritise notifications in a hypothesised power-saving mode. Notifications are perceived as the main functionality of the smartwatch, with $98 \%$ of participants ranking it as the most important feature [20]. Similarly, other studies report the notification functionality $[18,40]$ and the associated ability to access smartphone information inconspicuously $[6,23,25]$ as equally important, indicating that smartwatches play a different social role when compared to smartphones.

As the main perceived benefit of smartwatches is related to notifications, we designed a study to investigate the frequency and context of smartwatch notifications, and their associated usage sessions. We analyse a dataset collected from 307 unique users during the first half of 2016, and perform a quantitative analysis on how and when users interact with their smartwatches. We analyse the dataset in terms of usage session types based on both the source of the device use, and in terms of interaction styles. To the authors' knowledge, this dataset is the largest currently available on smartwatch usage.

\section{RELATED WORK}

While early smartwatch models faced significant technical limitations for general adoption (e.g., limited battery life [27]), these problems have since been reduced. Min et al. [20] studied the recharging habits and battery use of smartwatches, and concluded that a modern smartwatch can easily maintain functionality for a period of at least 24 hours, usually more, depending on the user. The participants were not unanimously satisfied with the battery life of their smartwatches and 53\% experienced situations where the smartwatch ran out of battery more than once per week. In 
this paper we focus on the way smartwatches are used, and not on the technical capabilities of the devices.

\section{Purpose and Usage of Smartwatches}

Wu et al. [39] conducted a survey of 212 participants, and drew conclusions on the different factors that impact consumer's acceptance of the smartwatch. Neither ease of use nor gender have impact on the acceptance of the technology, as people have already accustomed to handling wristwatches. The key requirement is result demonstrability $[21,36]$, indicating that the outcome of smartwatch use should be able to be both communicated and observed. A smartwatch can be used as a simple extension of a smartphone, where the benefits of its use need to be quantifiable, or as a standalone device/platform for an application, where the benefits are implicit as long as the application(s) are useful to the consumer.

Smartwatches also enable inconspicuous use, compared to smartphones, which can release the social tension caused by frequent smartphone use [23,25]. Additionally, the nature of a persistently carried device on one's wrist can reduce the problem of user availability [3,30]. Yalçin [40] reports survey results regarding prioritising notifications on smart devices and notes that, similar to smartphones, users attend to notifications from specific application categories, such as communication or calendar applications, and tend to dismiss notifications from other types of applications, such as games. PrefMiner is an application developed by Mehrotra et al. [19] for creating personal notification preferences, but is limited to smartphones. Weber et al. [38] studied users' notification preferences in multi-device environments with over $50 \%$ of their participants wanting to receive notifications via their smartwatch instead of their larger devices.

\section{Applications of Smartwatches}

Wearable devices have become an important tool in solving health problems [22] as they provide various applications for monitoring energy expenditure, hence promoting physical activity amongst users [5]. In particular, the gamification aspect of smartwatch applications encourage improvement or keep up the healthy lifestyle by rewarding the users for achieving the daily steps or exercises goals [16].

Smartwatches may collect biological, environmental, and behavioural information about user's activity due to constant contact with skin [15]. Smartwatches are also likely to be carried in-person throughout the day and do not have the issue of being forgotten somewhere, or being in a state where the user is not necessarily alerted of incoming messages or notifications.

Researchers also studied detection of eating [7,26,33], smoking [31,33], drinking coffee [33], and giving a talk [33]. Findings suggest that being able to detect human activities might help in mitigating bad habits, such as drinking too much coffee, smoking, or skipping a meal [33], and argue that detection of user activities might be useful in determining the optimal time for interrupting the user. For example, the user should not be interrupted while typing or writing, but could be interrupted while drinking coffee or smoking [33].

\section{Micro-usage and Interactions}

The literature contains relevant work on user's behaviour on both smartphones and smartwatches. As the smartwatch is often used in conjunction with the smartphone of the user, its usage patterns influence the way smartwatches are used. Smartphone usage can be classified into glance, review, and engage categories [2], ranging from short interactions in which only the lock screen is queried for the latest notifications, to long usage sessions in which multiple applications are used.

Ferreira et al. [9] introduced the term application microusage, describing the brief bursts of application interaction: "approximately $40 \%$ of application launches last less than 15 seconds and happen most frequently when the user is at home and alone" [9]. Smartphone usage is typically short, with Yan et al. [41] finding that 50\% of mobile phone engagement lasts less than 30 seconds. Van Berkel et al. [35] distinguish between continued and new usage sessions. Their results show that for the majority of cases in which people unlock their phone, they intend to start a new objective as opposed to continuing a previous one. Previous work also briefly looks at the types of sessions and interactions on smartwatches, and both Min et al. [20] and Gouveia et al. [13] report users habitually engaging in brief usage sessions.

Notifications are a critical component of smartphone interaction, with users receiving an average of 63.5 notifications per day according to study by Pielot et al. [24]. Most notifications are checked by the user within a short time interval, with messages and emails generating majority of notifications [24]. New ways in which users will operate their combined smartphone and smartwatch are being actively explored (e.g., a diabetes diary toolset [1]), as well as explorations on which device is more suitable for which interaction (e.g., driver engagement to notifications [12]).

Although notifications are inherently disruptive and distractive [29], users place value on receiving notifications from sources important to them. For instance, Sahami Shirazi et al. [29] conducted a large-scale assessment of mobile notifications and report that users value notifications depending on their source: messages and notifications about people and events are more important, in line with the survey results of Yalçin [40].

\section{DATASET}

Our smartwatch dataset was collected in-the-wild with an application available on Google Play Store called Insight 4 Wear ${ }^{1}$, introduced in [28]. The user accepts the provided enduser license agreement to allow the application to store the

\footnotetext{
${ }^{1}$ http://insight4wear.com
} 
information locally and periodically (every three hours), check for Wi-Fi connectivity, and upload the logged data to the server. All data is anonymised to respect the user's privacy and no contact information is stored. The users are all anonymous and only device identification numbers (Android device ID) are stored. Due to the anonymity of our users, we are not able contact them. The demographic distribution of the users (provided by Google Play Store) shows $44.72 \%$ of the users are from North America (US and Canada), 26.13\% from Central Europe (UK, Germany, Spain, France, Netherlands), 3.02\% from Australia, $2.51 \%$ from India, and $23.62 \%$ from elsewhere. Our analysis considers the following recorded data:

\section{Data}

Entries

Notification information

Time when the notification was displayed on

the device, and what application triggered it.

\section{Screen events}

When the screen was turned on, when the screen turned off.

\section{Device ID}

Table 1. Information about the dataset.

In total, 307 users contributed data. The data was collected between January 1st 2016 and July 15th 2016. 74.6\% of the users $(\mathrm{N}=229)$ had the application installed and logging for less than 30 days $(\mathrm{M}=7.09$, median $=4.02), 19.5 \%$ of the users $(\mathrm{N}=60)$ for a timespan between 30 and 90 days $(\mathrm{M}=$ 74.86 , median $=64.03)$, and $5.9 \%$ of the users $(\mathrm{N}=18)$ for more than 90 days $(\mathrm{M}=133.40$, median $=135.47)$. We were able to collect manufacturer and model details from 267 devices (Table 2).

\section{Application Categories}

Each notification contains the package name of the triggering application, which is a unique identifier set when the developer uploads the application to Google's Play Store. This package name is stored with each arriving notification. Thus, we were able to infer the applications category of each notification in our dataset by cross-referencing with the Play Store. In addition, we followed the recommendation by Brown et al. [4], and further grouped applications into more general categories (Table 3).

\begin{tabular}{llr} 
Device Model & Manufacturer & Devices \\
\hline Moto 360 & Motorola & 83 \\
HUAWEI WATCH & HUAWEI & 49 \\
SmartWatch 3 & Sony & 39 \\
G Watch R & LG & 36 \\
LG Watch Urbane & LG & 23 \\
G Watch & LG & 12 \\
ASUS ZenWatch 2 & ASUS & 9 \\
Gear Live & Samsung & 9 \\
ASUS ZenWatch & ASUS & 5 \\
Samsung Gear 2 & Samsung & 2 \\
Unknown & Unknown & 40 \\
\hline
\end{tabular}

Table 2. Distribution of device models.

General Category Application Categories [4]

\begin{tabular}{|c|c|}
\hline Communication & Communication \\
\hline $\begin{array}{l}\text { Productivity \& } \\
\text { Admin }\end{array}$ & $\begin{array}{l}\text { Productivity, Tools, Education, Business, } \\
\text { Books and Reference, Finance, } \\
\text { Personalization }\end{array}$ \\
\hline Health & Lifestyle, Health and Fitness, Medical \\
\hline Internet \& Social & $\begin{array}{l}\text { Entertainment, News and Magazines, } \\
\text { Sports, Social }\end{array}$ \\
\hline Maps and Travel & $\begin{array}{l}\text { Travel and Local, Weather, Transportation, } \\
\text { Shopping }\end{array}$ \\
\hline Media & $\begin{array}{l}\text { Music and Audio, Photography, Media and } \\
\text { Video }\end{array}$ \\
\hline Games & All categories of Games \\
\hline Other & Unspecified or unlisted in the Play Store \\
\hline
\end{tabular}

Table 3. Application generic categorisation.

\section{Smartwatch Data Pre-processing}

Android Wear smartwatches allow interaction through touch or voice commands. The screen can be turned on by touch, by moving one's wrist, or by incoming notifications as long as the smartwatch is already in a specific position (the screen facing upwards) or if the light sensor detects a light source towards the screen. This makes it challenging to programmatically infer intentional interaction. We use similar methodology as Gouveia et al. [13] to infer usage sessions where we consider the screen ON and OFF events on the device.

To infer interactions and usage sessions from the dataset, we combine screen use data (indicating when the screen was turned on) and notification data (indicating when a new notification has arrived on the smartwatch). We begin by cross-referencing the arrived notifications with the screen use data.
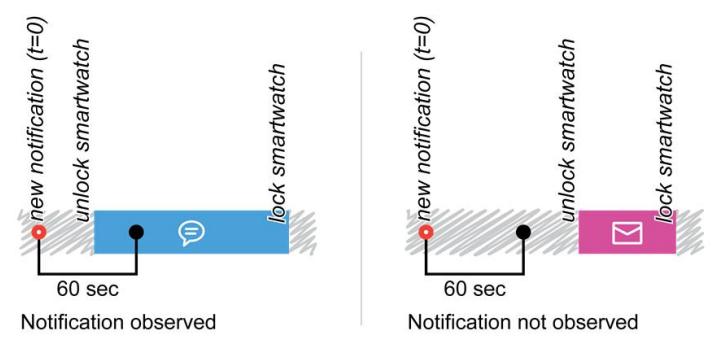

Figure 1. Labelling a notification as observed or not observed.

Figure 1 illustrates the two different scenarios: a notification is either observed or not observed following a specified time window. We apply a $60 \mathrm{sec}$. window to each notification, after which we consider the particular notification not observed if no screen events were recorded. The threshold was determined based on previous work [29], that showed that roughly $60 \%$ of interactions with notifications occurs in the first 60 seconds, followed by a very long tail for remaining interactions. Ultimately, for each notification we include: user ID, source application, time, label (observed or not observed), and session length (if available). 
Next we analyse each of the logged usage sessions using a $60 \mathrm{sec}$. window (Figure 2) to determine if a usage session was notification initiated (reactive) or user initiated (proactive). For each usage session we include: user ID, start time, duration, usage label (user vs. notification initiated), and source application of the notification (if available).

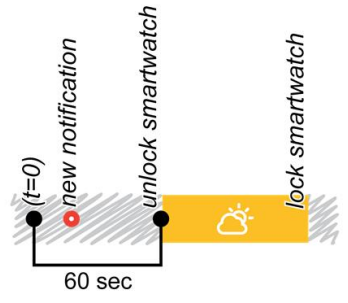

Notification initiated (reactive)

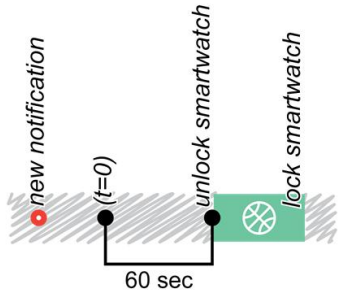

User initiated (proactive)
Figure 2. Labelling each session as notification initiated or user initiated.

The dataset contains some known outliers. For example, usage session where the screen is turned on for a long period of time are instance where the devices are likely to be charging. To prevent these erroneous entries from skewing the data, we apply a low-pass filter of 5 minutes. We base the threshold on two earlier studies $[8,41]$ on smartphone session lengths, where the session length ranges from ten seconds to four minutes. We argue that removing the entries over this threshold discards sessions where the user was not aware of the device's screen being on, or sessions where the user did not interact with the device regardless of the screen being on. Additionally, the sessions analysed by past literature (for smartphones) report continuous usage sessions, consisting of several individual sessions, tend to not exceed five minutes on average [34,35].

Observing the distribution of usage session length (where the notification was not ignored or missed, $\mathrm{N}=328,587$ ), we noted that the median session length is exactly 5 seconds and that $22.1 \%$ of all the sessions have this exact length $(\mathrm{N}=$ $72,786, \mathrm{M}=8.63)$. These values are also visible in Figure 10. This is a hardcoded delay when the screen turns on and off without any further user interaction. Therefore, we label all sessions lasting five seconds or less as "peek", and those lasting longer than five seconds as "interaction".

Because the preferred smartwatch features broadly fall under "functionality" and "notifications" [16,20], we divide usage sessions into a) proactive sessions (where the smartwatch was used to glance at screen for time unprompted), and b) reactive sessions (sessions where the device use was due to an arriving notification).

Our pre-processing gives rise to two datasets. Firstly, a dataset of 798,423 usage sessions labelled by session type (peek vs. interaction) and session initiator (notification vs. user). The distribution of usage sessions to these categories is shown in Figure 3.

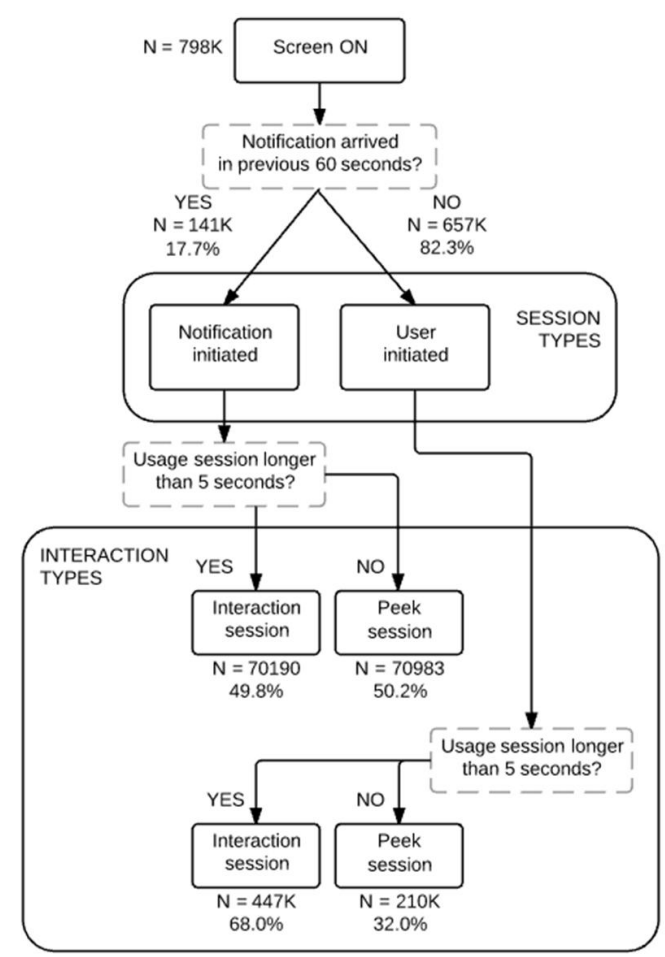

Figure 3. The categorisation of usage sessions.

Secondly, a dataset of 2,801,082 entries containing the notifications that arrived on the smartwatch, labelled according to the users' response: observed or not observed, along with the length of the resulting usage session and the delay between the notification arriving and the beginning of the session. This distribution is shown in Figure 4.

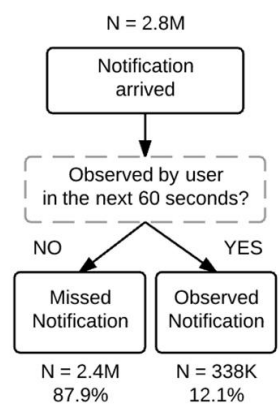

Figure 4. The categorisation of arriving notifications.

Both smartwatch datasets contain further data on user, time, application, and application category.

\section{Comparison to Smartphone Dataset}

From four previously published studies [10,14,35,37], we aggregate a dataset of 347,576 smartphone screen entries, from 445 unique users between February 2014 and May 2016, which we then transform into 120277 unique usage sessions. Each session has an associated session length $(\mathrm{M}=$ 229.34 seconds), and the daily session count $(M=60.08)$ for 
that user on that particular day. This dataset is used to draw quantified comparisons between usage sessions on smartwatches and smartphones. There was no information in these datasets regarding the arrival of notifications or used applications so analysis on these aspects was not performed.

\section{ANALYSIS AND RESULTS}

We refer to usage session or session to describe instances where the smartwatch was used (the screen was turned on). We identify two interaction types:

- Peek, a brief usage session where the device is used for five seconds or less. $(\mathrm{N}=517,989)$.

- Interaction, a longer usage session lasting for more than five seconds. $(\mathrm{N}=280,434)$.

\section{And two session types:}

- User-initiated (proactive): A usage session that does not follow a notification arriving on the device, indicating that the user chose to interact without being prompted. $(\mathrm{N}=657,250)$.

- Notification-initiated (reactive): A usage session that follows a notification arriving on the device within the previous 60 seconds, indicating that the user was prompted to use the device due to the notification. $(\mathrm{N}=141,173)$.

We use a Chi-Square test to analyse how usage sessions and notifications are divided throughout the day. Unsurprisingly, the results show that both sessions $\left(\chi^{2}=254,280, \mathrm{p}<0.05\right)$ and notifications $\left(\chi^{2}=452,700, \mathrm{p}<0.05\right)$ are unevenly spread across the hours, mostly focused during $8 \mathrm{am}-10 \mathrm{pm}$. Users who had logged data from at least seven days had an average of 142.1 usage sessions (5,107 days, 725,548 sessions) per day. The usage sessions from smartwatch and smartphone datasets are divided across day according to Figure 5. The increased use of the smartwatch during daytime (8am to $8 \mathrm{pm})$ is clearly visible in the distribution of the usage sessions.

Next we describe the results of our analysis on the different interaction and session types in terms of hourly frequency during a day, followed by other conditions, such as application category.

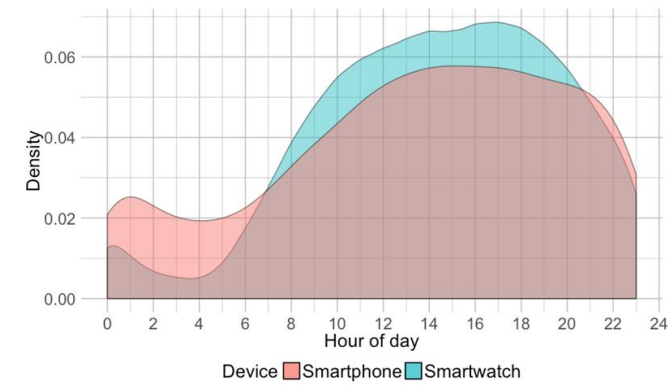

Figure 5. Distribution of sessions throughout the day.

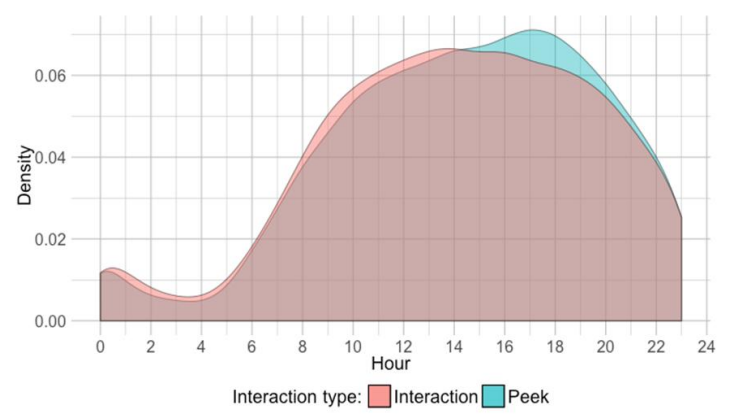

Figure 6. Session density on smartwatch throughout the day according to interaction type.

\section{Usage Sessions on Smartwatches}

We analyse the frequency of sessions by comparing the

"peek" $(\mathrm{N}=517,989)$ and "interaction" $(\mathrm{N}=280,434)$ categories using a Chi-Square test for both hourly granularity, and for period of the day (divided into night, morning, afternoon, and evening). Both tests are significant $(\mathrm{p}<0.05)$ for the two different categories, hour of day $\left(\chi^{2}(\right.$ hour $\left.)=1192.9\right)$ and period of day $\left(\chi^{2}(\right.$ period $\left.)=611.9\right)$, indicating that the time and period of the day result in different type of device usage. In the later hours of the day (from $2 \mathrm{pm}$ to $10 \mathrm{pm}$ ), users are more likely to engage in brief peek sessions, rather than in a longer interaction session (Figure 6)

We also analyse the frequency of sessions according to whether they were user initiated $(\mathrm{N}=657,250)$ or notification initiated $(\mathrm{N}=141,173)$. Two Chi-Square tests considering hour of day and period of the day gave significant results $(\mathrm{p}<0.05) ; \chi^{2}$ (hour) $=203,820$ and $\chi^{2}($ period $)=164,940$ for user initiated sessions; $\chi^{2}$ (hour $)=$ 51,605 and $\chi^{2}$ (period) $=41,313$ for notification initiated sessions. Finally, a Chi-Square test between the two conditions (user initiated and notification initiated) across the hours of the day was significant $\left(\chi^{2}=898.09, \mathrm{p}<0.05\right)$.

These results indicate that the two types of sessions vary significantly during the day (Figure 7), and across each other. Specifically, we observe that during the morning and

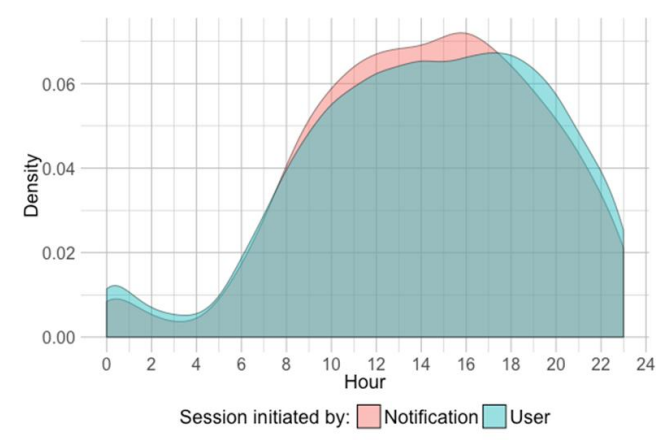

Figure 7. Smartwatch session density throughout the day according to session type. 
afternoon (between $8 \mathrm{am}$ and $5 \mathrm{pm}$ ) a device use is more likely to be initiated by an arriving notification than by the user's own volition.

Next we consider how session duration varies throughout the day. A two-way ANOVA shows that the interaction effect of how a session is initiated (user vs. notification) and hour of the day significantly affect session duration $(\mathrm{F}=25.56, \mathrm{p}<$ $0.05)$, as well as both the main effects $(\mathrm{F}$ (hour) $=161.66$, $\mathrm{F}($ session type $)=4419.77, \mathrm{p}<0.05)$.

The findings show that the length of the smartwatch session fluctuates during the day, and sessions tend to be longer during the night (Figure 9). The smartphone sessions show less fluctuations, and the mid-day sessions are the shortest. Also, smartwatch sessions initiated by notifications are longer: the mean length for notification-initiated sessions is 10.67 seconds, and 7.94 seconds for user-initiated (Figure 9 and Figure 10). The differences of the session length distributions between the two smartwatch conditions are clear, as user initiated use is often shorter than, or exactly five seconds, indicating brief glances at the device. Note that sessions less than five seconds in length indicate the user interacting with the device followed by actively turning off the screen, which also happens more frequently for userinitiated sessions. Additionally, when comparing the session lengths between the two devices, smartphone sessions are clearly longer duration.

Next, we consider the hourly frequency of users either observing (notification followed by device use), or not observing a notification (no device use after an arriving notification). A Chi-Square test was statistically significant $\left(\chi^{2}=31.801, \mathrm{p}<0.05\right)$ indicating that users are more likely to observe the arrival of a notification during different times of day. Specifically, users are more vigilant in the late afternoon ( $4 \mathrm{pm}$ to $6 \mathrm{pm}$ ) and less vigilant early in the morning or late in the evening. The hourly trend of observed notifications is very similar to the general trend of arriving notifications with a significant correlation between the two distributions $(\mathrm{t}=17.248, \mathrm{p}<0.05, \mathrm{r}=0.97)$. The distribution of notifications across the day is shown in Figure 11.

Finally, following the recommendations by Van Berkel et al. [35] and Soikkeli et al. [34], we verify the existence of continuous sessions consisting of multiple individual sessions within a certain time threshold $(\mathrm{T}=45$ seconds, as per [35]). This may represent cases where a user quickly and repeatedly uses their smartwatch, with short breaks in between, to complete a larger objective. For each session we look for a subsequent user initiated session within this time threshold recursively. We found that $81.1 \%$ of usage sessions do not satisfy this requirement, while $10.9 \%$ of cases consist of two consecutive sessions, and $9 \%$ consist of three or more (Figure 12). These results show the scattered nature of smartwatch use, and also show that with more frequent use continuous sessions with more than two individual sessions - the average length of the interaction in each individual session decreases.

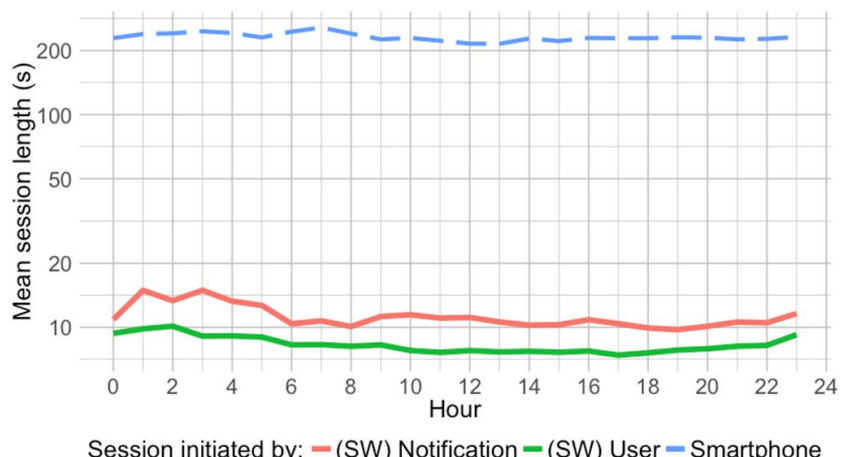

Figure 9. Mean session lengths throughout the day according to the smartwatch (SW) session type (red, green) and device type $($ Smartphone $=$ blue $)$.

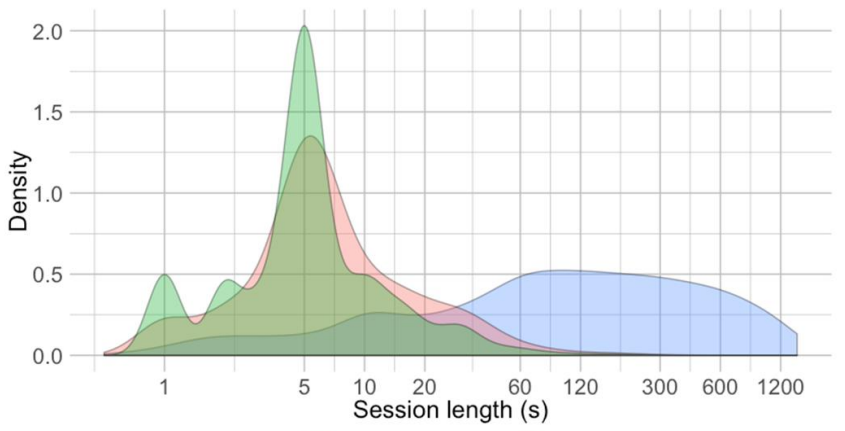

Session initiated by: $\square$ (SW) Notification $\square$ (SW) User $\square$ Smartphone

Figure 10. Overall density of session length distribution for both smartwatch (SW) session type (red, green) and device type $($ Smartphone $=$ blue $)$.
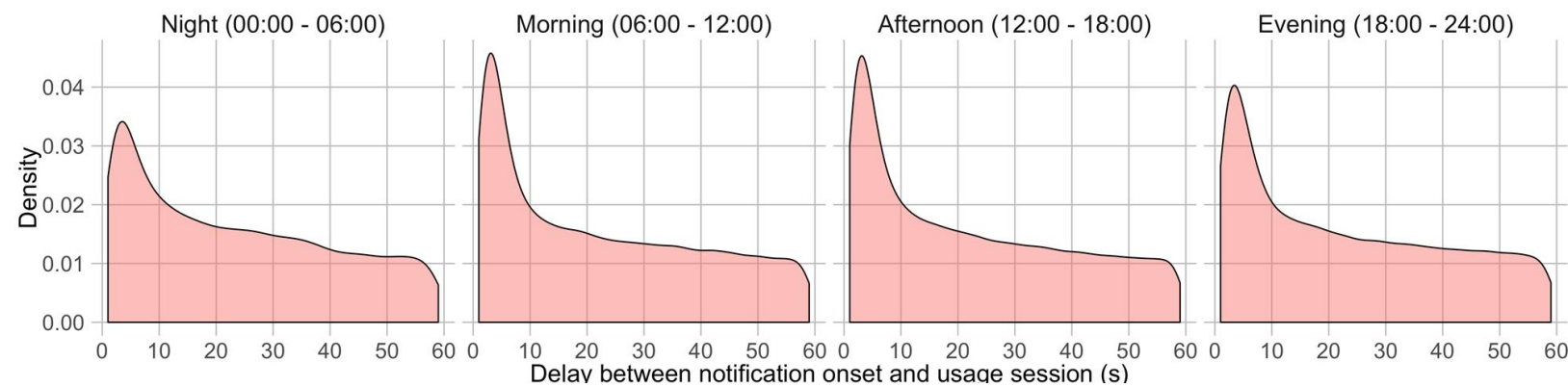

Figure 8. Delay between an arriving notification and the following usage session according to the period of day. 


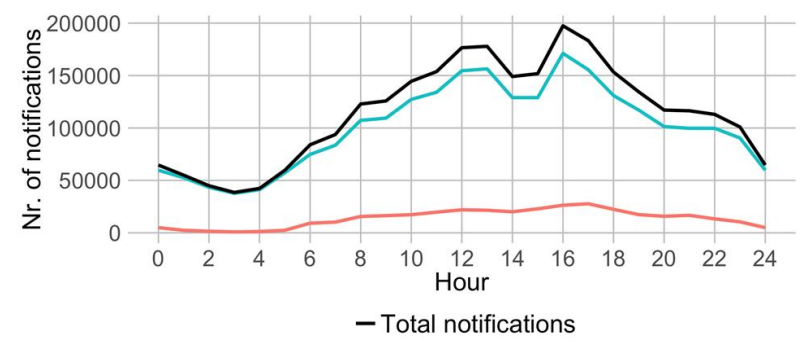

- Notification observed - Notification not observed

Figure 11. Distribution of arriving (black), observed (red), and not observed (blue) notifications throughout the day.

\section{Notifications and Application Categories}

In the collected dataset, most notifications are from five application categories: $38.5 \%$ from "Tools", $21.9 \%$ from "Travel and Local", $11.6 \%$ from "Other", 9\% from "Communication", and 6\% from "Health and Fitness". Only $9.4 \%$ of all notifications result in potential interaction sessions (peek or interaction sessions), and $19.7 \%$ of the notifications arrive when the device is already in use, or while charging.

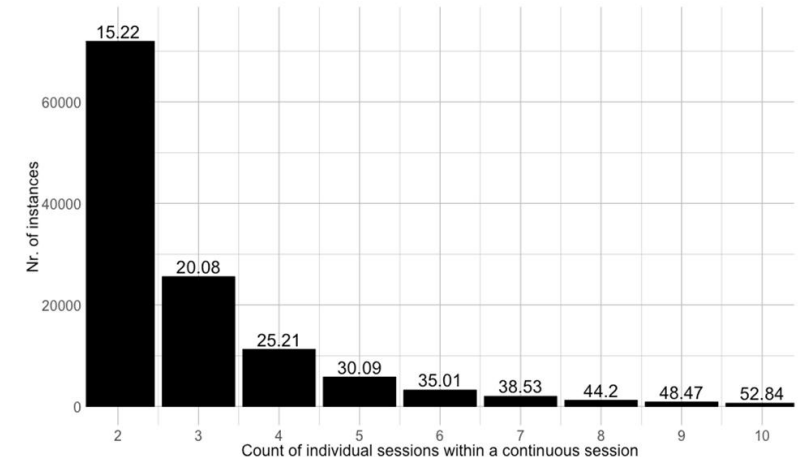

Figure 12. Multiple individual sessions make up a continuous session. Number above bar represents the mean duration (sec) of the continuous sessions (excluding breaks between use).

The mean delay between a notification arriving and the user taking action is 23 seconds (median $=20$ seconds), and the mean interaction session length is 17.89 seconds. The delay between the notification arriving and the start of a usage session was significantly different $(F(3,338,132)=43.83$, p $<0.05)$ across the different periods of the day. We chose not to test the difference in delay per application category, because the user has no way of knowing which application was responsible for the notification prior to observing the notification and starting new usage session. Users observe arriving notifications faster during daytime (6am to $6 \mathrm{pm}$ ) than during the evening and the night.

Of the notification-initiated sessions, $50.4 \%$ lasted equal or less than 5 seconds and are considered peek instances, while the remaining $49.6 \%$ were longer interaction instances.

To analyse the impact of application categories on session types, we ran a Chi-Square test over the two variables session type (interaction vs. peek) and application category. The results were significant $\left(\chi^{2}=7,124.9, \mathrm{p}<0.05\right)$ and can be seen in Figure 13. These results indicate that application category of an arriving notification has a significant impact on the resulting device use, in terms leading to interaction or being peeked at. Users are more likely to start an interaction session when the arriving notification is from e.g. "Communication" category, and more likely to peek (and not interact) when the notification is triggered by an application from "Maps and Travel" or "Health" category.

Next we look at the relationship between session length and application categories. We compare session length across both the general categories and the individual application categories (see Table 3). A statistically significant difference (one-way ANOVA) was found between session length and both the general category level $(\mathrm{F}(7,141165)=133, \mathrm{p}<$ $0.05)$ and application category level, $(\mathrm{F}(36,141136)=67.86$, $\mathrm{p}<0.05)$ respectively. Sessions initiated by "Communication" or "Other" notifications lead to longer session lengths than from e.g. "Maps and Travel". The results are visualised for the general categories in Figure 14.

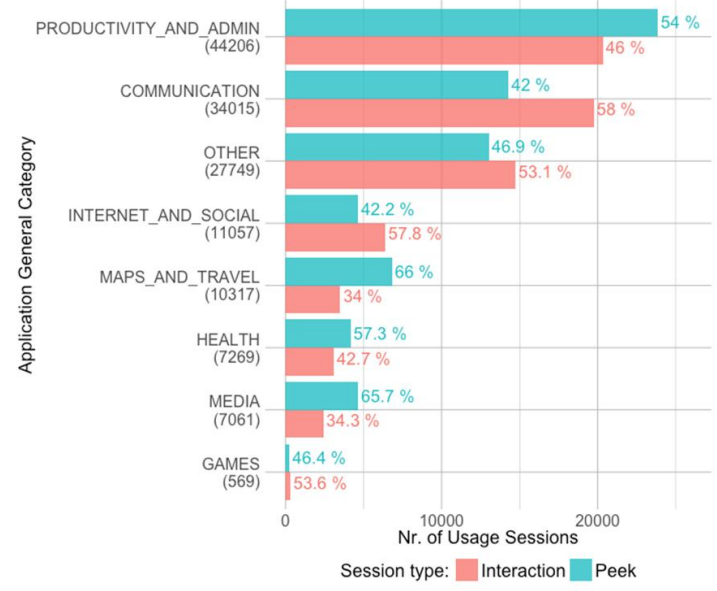

Figure 13. Interaction type per general application category.

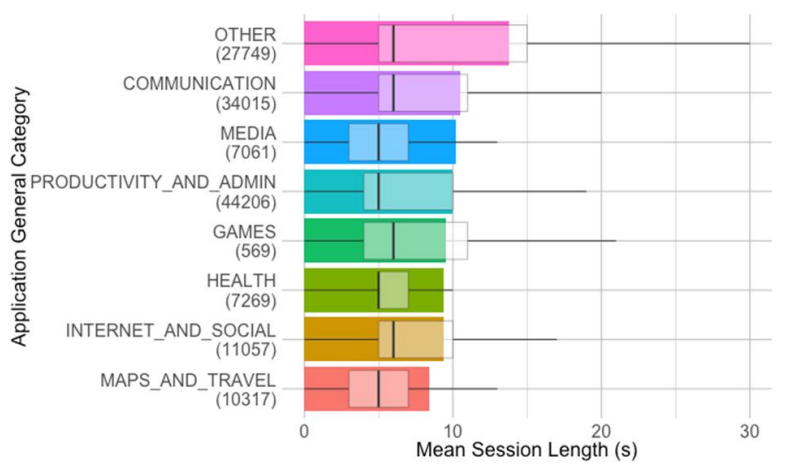

Figure 14. Mean (bar) and distribution (box) of session lengths for different general application categories.

Lastly, we analyse the distribution of usage sessions initiated by an arriving notification over the course of the day using both the general application categories and individual application categories. The results were significant for both 
general $\left(\chi^{2}=3,360.4, p<0.05\right)$ and individual $\left(\chi^{2}=9,795.6\right.$, $\mathrm{p}<0.05)$ application categories, indicating that time of day has an impact on the frequency of notification-initiated usage sessions from different application categories, as visualised in Figure 15. The distributions are normalised for each category (horizontally), so the visualisation shows that users prefer notification initiated use from "Communication" applications during the daytime (from $8 \mathrm{am}$ to $4 \mathrm{pm}$ ) over other periods and from "Internet and Social" applications during the evening ( $6 \mathrm{pm}$ to $10 \mathrm{pm})$.

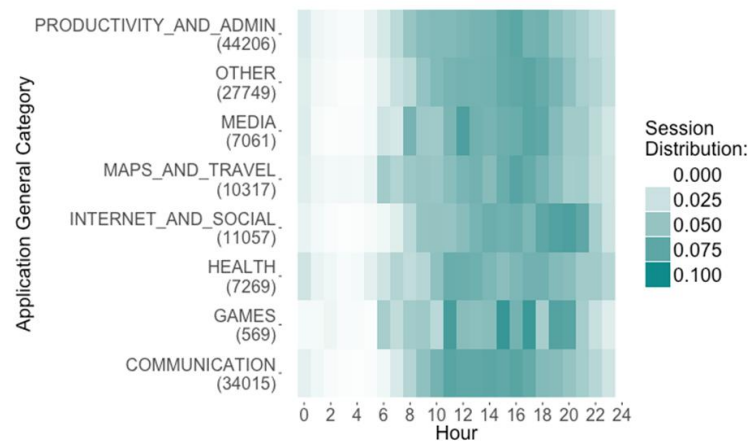

Figure 15: Notification initiated sessions according to the source (general category) of the arriving notification.

In conclusion, our results show that the nature of smartwatches leads to different type of use than with smartphones. The results of session lengths and daily counts between our smartwatch and smartphone datasets are visualised in Figure 16. The trend of shorter and more frequent sessions on smartwatches is clearly visible.

\section{DISCUSSION}

Given our analysis of a large-scale smartwatch usage dataset, we are now able to draw comparisons to previous findings on smartphone usage found in literature. To the authors' knowledge, our work is the first to report a large-scale quantitative investigation of smartwatch use in-the-wild. In addition, we consider how both internal factors (notifications) and external factors (time of day) affect use.

External factors in particular enable us to begin articulating the daily habits and activities of the users. Conversely, internal factors reveal interaction requests from the device in the form of arriving notifications. The combination of these two factors captures some of the underlying reason for smartwatch usage sessions, and offers some insight about the purpose of these devices, and their relationship to smartphones.

\section{Session Types}

We identify two session types, user initiated (proactive use) and notification initiated (reactive use) and show how the internal factor of requested attention impacts the frequency of the two different types (Figure 7).

The majority of sessions $(80.0 \%)$ are initiated by the user, with these user initiated sessions being on average shorter (mean of 7.94 seconds) than notification initiated sessions (mean of 10.67 seconds) (see Figure 9 and Figure 10), while sessions on smartphones are longer - Soikkeli et al. [34] report 4 minutes and 23 seconds on average and data from our aggregated datasets reports 3 minutes and 49 seconds.

Since the smartwatch also serves as a device used for timekeeping, a large portion of usage is expected to be peeks. Our results in Figure 3 show that about more than half the sessions $(65.2 \%)$ are peeks, for example to check for arriving notifications or new messages, even when no cue is presented to signal the arrival of a notification or a new message.

These results suggest that the habit of quick unprompted peeks (and micro-usage [9]) seems to carry from smartphones to smartwatches, and longer unprompted interaction sessions are less frequent. For smartphones, Banovic et al. [2] classify a median of $46.6 \%$ smartphone usage sessions as a glance session, which is their shortest classification on usage duration. We argue that for smartwatches, ease of access results in an increase in peeks, since the user does not need to go through the physical procedure of digging his or her pocket or bag for the device.

This ease of access is further highlighted by the fact that our smartwatch users had on average 142.1 sessions per day. The amount of daily sessions is higher than the previously reported values by Gouveia et al. (107 sessions per day) and Min et al. (95.6). This is an order of magnitude bigger than the respective values for smartphone use, as in our aggregated smartphone dataset we identified an average of 60.1 sessions per day. Van Berkel et al. [35] report differences in the total session lengths when taking into consideration continuous sessions consisting of multiple

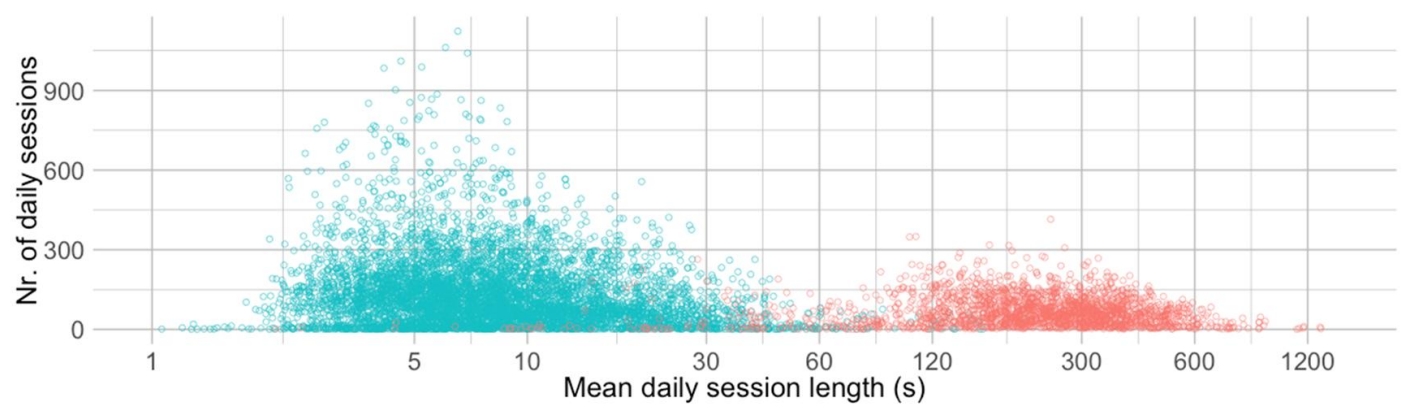

Figure 16. Distribution of daily usage sessions between smartwatches (blue) and smartphones (red). 
individual sessions, but for our smartwatch dataset the session lengths of the continuous sessions follow a different trend. When repeatedly initiating new usage sessions close to each other, the average length of each individual session is progressively reduced, indicating less interaction with the device and type of use that can even further be more associated to the (habits) of brief glances.

Multiple elements are likely to contribute to the differences in smartwatch and smartphone use. First, smartwatches are limited in their input capabilities, making these devices more suitable for consuming content rather than generating new content. Second, the typical placement of the smartwatch (on the wrist of the user) allows for quick interaction, explaining the higher number of daily interactions. Third, the smartwatch is likely to be used as a timekeeping device, adding to the number of (short) interactions. Last, the current user base of smartwatch users is most likely to be selfselected technology enthusiasts, therefore also explaining the high number of daily usage sessions.

User expectations with regards to notifications are different for different form factors, as was shown by Sahami Shirazi et al. [32]: calendar, VOIP, and messenger notifications rated as most important for smartwatch users. Figure 13 shows that the application categories of "Productivity and Admin" and "Communication" make up for the most number of usage sessions. This indicates that not only do users believe notifications from these applications are most important, but these applications actually occupy most of their smartwatch usage time.

\section{Impact of Application Types}

Next, we analyse the associations between different application types and smartwatch use in more detail. We found that both the frequency and length of a session are impacted by the application type, as shown in Figure 14 and Figure 15. The visualised data consists solely of sessions following a notification. This indicates that when a user's attention is requested, the source of request significantly impacts the resulting interaction. Similar to results reported by Sahami Shirazi et al. [29] - users are slower to respond and more likely to dismiss notifications from sources they do not deem important - the source of the notification on smartwatches also impact the resulting interaction. Notifications generated by different application types are more likely to prompt interaction sessions at different times of the day (Figure 15), result in interactions of different length (Figure 14), and also impact the session type (Figure $13)$.

This behaviour of selective use based on the source of the notification is similar to that observed on smartphones, with similarities reflect even the level of application category. Users are more likely to interact (usage of longer than five seconds) with the smartwatch when prompted by an application from "Communication", "Internet and Social", or "Other" general categories, and more likely to peek the smartwatch when prompted by an application in the
"Productivity and Admin", "Maps and Travel", "Media", and "Health" categories. These results match the survey results of previous work of Yalçin [40].

Our results also indicate the user can benefit from the ease of access provided by the wrist-worn smartwatch:

- As a tool for communication and social interaction, as indicated by the finding that users are more likely to interact when prompted by an application in the "Communication" or "Internet and Social".

- As a tool for navigation, quickly glancing for guidance or instructions, as indicated by fact that users are likely to spend less than five seconds using the smartwatch when prompted by a "Maps and Travel" application. Another corollary can be that these prompts are dismissed by the user.

The inherent nature of the same notifications being presented to both the user's smartwatch and smartphone prompts a similar style of interaction on both devices. This is reflected by the fact that the impact of notification source on smartwatch interaction is similar to that observed in the smartphone. Our results might give precedence to the idea that notifications that require interaction can be pushed to the smartwatch only if they are likely to be relevant for interaction methods available on the smartwatch. Applications that are more preferably handled on the bigger smartphone could be delivered there.

\section{Temporal Context}

The smartwatch also features some unique interaction possibilities. Smartphones have been criticized for their disruptive nature in social context, with users admitting they disapprove of mobile usage behaviour they display themselves [25]. Here, the smartwatch could potentially offer a more inconspicuous way of fulfilling these information needs. We measured the external factor of time and its impact on the types of usage sessions. For most of the day, except afternoon hours, the frequency of peek and interaction sessions are similar, as visualised in Figure 6. The main differences between the conditions occur during the afternoon hours. It is also shown that most notifications are observed by the user during noon (lunch hours) and late afternoon (around 4). However, considering that overall distribution of observed notifications and distribution of notifications over the day (Figure 11) correlate strongly, indicating that the time of the day itself has no significant impact on the likelihood of a notification going unnoticed, as more notifications also arrive during these time periods.

The low impact of time on the ratio of observed notifications is an indicator of one of the benefits of the smartwatch, as the user's availability is not hindered at different periods of the day, as it occasionally is when using solely a smartphone $[3,30]$. Further, the user is as likely to be reached by a notification when time is sparse as he is in a more tranquil period of the day. This is also in line with the nature of the 
smartwatch, as the device itself is commonly present for the user.

Smartphones on the other hand can be located in a pocket or on a table, outside of reach for the user and with the screen not always visible. Even the physical cues such as sound or vibration generated by the smartphone are not always noticed by the user. On the smartwatch, as long as worn by the user, these problems are less likely to occur and the cues are more likely to be observed.

The smartwatch as a covert device offering information to the user also deals with some of the social issues brought up by previous work $[23,25]$ regarding frequent smartphone usage. The results in Figure 8 and the median delay $(20.0$ seconds) of all notifications show that users interact with notifications on smartwatches faster than with smartphones, as per Sahami Shirazi et al. [29] where the overall median was not reported, but where $50 \%$ of the notifications were interacted with within 30 seconds. Figure 8 also shows that most notifications lead to usage sessions swiftly (in less than 10 seconds) and the delay is even shorter during morning and afternoon hours. These results indicate that when used in tandem with a smartphone, a smartwatch offers the same functionalities for receiving information, in a quicker and more convenient way. This quantified result is in tandem with the user preferences reported by Weber et al. [38].

Figure 16 summarises our overall findings. Analysing smartwatch use over a period of six months and comparing it to a dataset of smartphone use gathered during two years, the differences in usage between these two device types become evident.

\section{Limitations}

During the analysis, we identified potential outliers that were erroneous as the result of the data collection tool. We use screen $\mathrm{ON}$ data to infer smartwatch usage (e.g., charging, user moving arm in a certain position), and therefore some false positives may have been included in the dataset, regardless of our attempts to discard such outliers. Given the size of dataset that was analysed, we are confident that these outliers did not systematically bias our results. Furthermore, our dataset consists only of Android Wear smartwatches. Hence, further research that investigate smartwatch usage with other operating systems is needed.

\section{CONCLUSION}

Our analysis looks at a large sample of logged smartwatch use from the first half of 2016 and an aggregated dataset of smartphone use between 2014 and 2016. The aim of the analysis is to increase our understanding of how smartwatches are used in-the-wild, and particularly compare it to smartphone usage.

Our results indicate that the behaviour of smartwatch usage bears resemblance to regular wristwatch usage. Brief and frequent usage sessions are preferred, with the user most likely switching to their smartphone for longer sessions involving the creation of new content as opposed to the consumption of content. We introduce a new term to describe these short usage sessions, peeks, in which the smartwatch is used for only five seconds or less.

We found that different application categories result in different types of usage. Similarly, the source of interaction changes depending on the time of day, suggesting a relationship to users' availability.

In an attempt to answer our earlier question - Should we be designing smartwatches or smartphone extensions? - we observe that the industry is taking steps towards the former. Companies such as LG and Samsung have recently aimed to release more powerful smartwatches aimed for standalone use $[11,17]$. These new and more powerful models do benefit from the point of increased availability. We expect that increased network and cellular availability and battery life will make the user less reliant on their smartphone.

Ultimately, our analysis shows that current smartwatches are used more frequently than smartphones, and that smartwatches are used in ways that have not been observed in the analysis of smartphone usage. These results are based on comparing datasets collected both on smartwatches and smartphones. Therefore, it can be argued that standalone smartwatches could build on these unique aspects. Furthermore, we find that user behaviour with regards to notification and application content is similar across both types of devices. We interpret this to indicate that users still prioritise the same type of content, while the method of interaction is adjusted to the unique characteristics of the smartwatch.

Based on our findings, we argue that there is room for smartwatches to be extended by creating novel interaction methods, something for example explored in [18]. However, we note that the type of content and applications that users will want to access is likely to remain the same, and users are likely to react similarly to notifications on their smartwatches. Hence, designing applications that leverage the simple interactions available on the smartwatch instead of relying on more complex input methods can significantly extend the benefits of using smartwatch in tandem with the smartphones, or as standalone devices.

\section{ACKNOWLEDGMENTS}

Anonymised

\section{REFERENCES}

1. Eirik Årsand, Miroslav Muzny, Meghan Bradway, Jan Muzik and Gunnar Hartvigsen. 2015. Performance of the First Combined Smartwatch and Smartphone Diabetes Diary Application Study. Journal of Diabetes Science and Technology 9, 3, 3556-563. http://dx.doi.org/10.1177/1932296814567708

2. Nikola Banovic, Christina Brant, Jennifer Mankoff and Anind Dey. 2014. ProactiveTasks: The Short of Mobile Device Use Sessions. In Proceedings of the 16th International Conference on Human-computer Interaction 
with Mobile Devices \&\#38; Services, ACM, 243-252. http://dx.doi.org/10.1145/2628363.2628380

3. Matthias Böhmer, Christian Lander, Sven Gehring, Duncan D. P. Brumby and Antonio Krüger. 2014. Interrupted by a Phone Call: Exploring Designs for Lowering the Impact of Call Notifications for Smartphone Users. In Proceedings of the 32Nd Annual ACM Conference on Human Factors in Computing Systems, ACM, 3045-3054. http://dx.doi.org/10.1145/2556288.2557066

4. Barry Brown, Moira McGregor and Donald McMillan. 2014. 100 Days of iPhone Use: Understanding the Details of Mobile Device Use. In Proceedings of the 16th International Conference on Human-computer Interaction with Mobile Devices \& Services, ACM, 223-232. http://dx.doi.org/10.1145/2628363.2628377

5. Andreas Bulling, Ulf Blanke and Bernt Schiele. 2014. A Tutorial on Human Activity Recognition Using Body-worn Inertial Sensors. ACM Comput. Surv. 46, 3, I33:1-33:33. http://dx.doi.org/10.1145/2499621

6. Marta M. E. Cecchinato, Anna A. L. Cox and Jon Bird. 2015. Smartwatches: The Good, the Bad and the Ugly? In Proceedings of the 33rd Annual ACM Conference Extended Abstracts on Human Factors in Computing Systems, ACM, 2133-2138. http://dx.doi.org/10.1145/2702613.2732837

7. Yujie Dong, Jenna Scisco, Mike Wilson, Eric Muth and Adam Hoover. 2014. Detecting Periods of Eating During Free-Living by Tracking Wrist Motion. IEEE Journal of Biomedical and Health Informatics 18, 4, I1253-1260. http://dx.doi.org/10.1109/JBHI.2013.2282471

8. Hossein Falaki, Ratul Mahajan, Srikanth Kandula, Dimitrios Lymberopoulos, Ramesh Govindan and Deborah Estrin. 2010. Diversity in Smartphone Usage. In Proceedings of the 8th International Conference on Mobile Systems, Applications, and Services, ACM, 179-194. http://dx.doi.org/10.1145/1814433.1814453

9. Denzil Ferreira, Jorge Goncalves, Vassilis Kostakos, Louise Barkhuus and Anind A. K. Dey. 2014. Contextual Experience Sampling of Mobile Application Micro-Usage. In International Conference on Human-Computer Interaction with Mobile Devices and Services, ACM, 91100. http://dx.doi.org/10.1145/2628363.2628367

10. Denzil Ferreira, Vassilis Kostakos, Alastair A. R. Beresford, Janne Lindqvist and Anind A. K. Dey. 2015. Securacy: An Empirical Investigation of Android Applications' Network Usage, Privacy and Security. In Conference on Security and Privacy in Wireless and Mobile Networks, ACM, 11:1-11:11. http://dx.doi.org/10.1145/2766498.2766506

11. First five things to know about the LG Watch Urbane 2nd Edition LTE. Retrieved August 31, 2016 from http://www.androidcentral.com/
12. Wayne W. C. Giang, Liberty Hoekstra-Atwood and Birsen Donmez. 2014. Driver Engagement in Notifications: A Comparison of Visual-Manual Interaction between Smartwatches and Smartphones. Proceedings of the Human Factors and Ergonomics Society Annual Meeting 58, 1, I2 161-2165. http://dx.doi.org/10.1177/1541931214581454

13. Rúben Gouveia, Fábio Pereira, Evangelos Karapanos, Sean S. A. Munson and Marc Hassenzahl. 2016. Exploring the Design Space of Glanceable Feedback for Physical Activity Trackers. In Proceedings of the 2016 ACM International Joint Conference on Pervasive and Ubiquitous Computing, ACM, 144-155. http://dx.doi.org/10.1145/2971648.2971754

14. Simo Hosio, Denzil Ferreira, Jorge Goncalves, Niels van Berkel, Chu Luo, Muzamil Ahmed, Huber Flores and Vassilis Kostakos. 2016. Monetary Assessment of Battery Life on Smartphones. In Conference on Human Factors in Computing Systems, ACM, 1869-1880. http://dx.doi.org/10.1145/2858036.2858285

15. Emil Jovanov. 2015. Preliminary analysis of the use of smartwatches for longitudinal health monitoring. In Annual International Conference of the IEEE Engineering in Medicine and Biology Society, IEEE, 865-868. http://dx.doi.org/10.1109/EMBC.2015.7318499

16. Martin Kracheel, Walter Bronzi and Hamed Kazemi. 2014. A Wearable Revolution: Is the smartwatch the next small big thing? IT ONE Magazine 2014 7, 18-19.

17. LG launches Watch Urbane 2nd Edition with LTE connectivity, higher-resolution display. Retrieved August 31, 2016 from http://www.androidcentral.com/lg-launcheswatch-urbane-2nd-edition-lte-connectivity-higherresolution-display

18. Janosch Maier and Wolfgang Wörndl. 2015. Smartwatch Interaction - More than just Notifications. In Mensch und Computer 2015 - Workshopband Anette Weisbecker, Michael Burmester and Albrecht Schmidt (eds.). De Gruyter Oldenbourg, 299-303.

19. Abhinav Mehrotra, Robert Hendley and Mirco Musolesi. 2016. PrefMiner: Mining Users Preferences for Intelligent Mobile Notification Management. In ACM International Joint Conference on Pervasive and Ubiquitous Computing, ACM, 1223-1234, http://dx.doi.org/ $10.1145 / 2971648.2971747$

20. Chulhong Min, Seungwoo Kang, Chungkuk Yoo, Jeehoon Cha, Sangwon Choi, Younghan Oh and Junehwa Song. 2015. Exploring Current Practices for Battery Use and Management of Smartwatches. In Proceedings of the 2015 ACM International Symposium on Wearable Computers, ACM, 11-18. http://dx.doi.org/10.1145/2802083.2802085

21. Gary G. C. Moore and Izak Benbasat. 1991. Development of an Instrument to Measure the Perceptions of Adopting an Information Technology Innovation. 
Information Systems Research 2, 3, I192-222. http://dx.doi.org/10.1287/isre.2.3.192

22. Bobak B. J. Mortazavi, Mohammad Pourhomayoun, Gabriel Alsheikh, Nabil Alshurafa, Sunghoon S. I. Lee and Majid Sarrafzadeh. 2014. Determining the Single Best Axis for Exercise Repetition Recognition and Counting on SmartWatches. In International Conference on Wearable and Implantable Body Sensor Networks, IEEE, 33-38. http://dx.doi.org/10.1109/BSN.2014.21

23. Leysia Palen, Marilyn Salzman and Ed Youngs. 2000. Going Wireless: Behavior \& Practice of New Mobile Phone Users. In Proceedings of the 2000 ACM Conference on Computer Supported Cooperative Work, ACM, 201-210. http://dx.doi.org/10.1145/358916.358991

24. Martin Pielot, Karen Church and Rodrigo de Oliveira. 2014. An In-situ Study of Mobile Phone Notifications. In Proceedings of the 16th International Conference on Human-computer Interaction with Mobile Devices \& Services, ACM, 233-242. http://dx.doi.org/10.1145/2628363.2628364

25. Lee Rainie and Kathryn Zickuhr. 2015. Americans' Views on Mobile Etiquette. 1-39.

26. Raul R. I. Ramos-Garcia and Adam A. W. Hoover. 2013. A Study of Temporal Action Sequencing During Consumption of a Meal. In Proceedings of the International Conference on Bioinformatics, Computational Biology and Biomedical Informatics, ACM, 68:68-68:75. http://dx.doi.org/10.1145/2506583.2506596

27. Reza Rawassizadeh, Blaine B. A. Price and Marian Petre. 2015. Wearables: Has the Age of Smartwatches Finally Arrived? Communications of the ACM 58, 45-47. http://doi.acm.org/10.1145/2629633 http://dx.doi.org/10.1145/2629633.

28. Reza Rawassizadeh, Martin Tomitsch, Manouchehr Nourizadeh, Elaheh Momeni, Aaron Peery, Ulanova Liudmila and Michael Pazzani. 2015. Energy-Efficient Integration of Continuous Context Sensing and Prediction into Smartwatches. Sensors 15, I22616 - 22645. http://dx.doi.org/10.3390/s150922616

29. Alireza Sahami Shirazi, Niels Henze, Tilman Dingler, Martin Pielot, Dominik Weber and Albrecht Schmidt. 2014. Large-scale Assessment of Mobile Notifications. In Proceedings of the SIGCHI Conference on Human Factors in Computing Systems, ACM, 3055-3064. http://dx.doi.org/10.1145/2556288.2557189

30. Antti Salovaara, Antti Lindqvist, Tero Hasu and Jonna Häkkilä. 2011. The Phone Rings but the User Doesn't Answer: Unavailability in Mobile Communication. In Proceedings of the 13th International Conference on Human Computer Interaction with Mobile Devices and Services, ACM, 503-512. http://dx.doi.org/10.1145/2037373.2037448
31. Philipp P. M. Scholl and Kristof K. V. Laerhoven. 2012. A Feasibility Study of Wrist-Worn Accelerometer Based Detection of Smoking Habits. In International Conference on Innovative Mobile and Internet Services in Ubiquitous Computing, IEEE, 886-891. http://dx.doi.org/10.1109/IMIS.2012.96

32. Alireza A. S. Shirazi and Niels Henze. 2015. Assessment of Notifications on Smartwatches. In Proceedings of the 17th International Conference on Human-Computer Interaction with Mobile Devices and Services Adjunct, ACM, 11111116. http://dx.doi.org/10.1145/2786567.2794338

33. Muhammad Shoaib, Stephan Bosch, Hans Scholten, Paul P. J. M. Havinga and Ozlem O. D. Incel. 2015. Towards detection of bad habits by fusing smartphone and smartwatch sensors. In International Conference on Pervasive Computing and Communication Workshops, IEEE, 591-596. http://dx.doi.org/10.1109/PERCOMW.2015.7134104

34. Tapio Soikkeli, Juuso Karikoski and Heikki Hämmäinen. 2011. Diversity and End User Context in Smartphone Usage Sessions. In International Conference on Next Generation Mobile Applications, Services and Technologies, IEEE, 712. http://dx.doi.org/10.1109/NGMAST.2011.12

35. Niels van Berkel, Chu Luo, Theodoros Anagnostopoulos, Denzil Ferreira, Jorge Goncalves, Simo Hosio and Vassilis Kostakos. 2016. A Systematic Assessment of Smartphone Usage Gaps. In Conference on Human Factors in Computing Systems, ACM, 4711-4721. http://dx.doi.org/10.1145/2858036.2858348

36. Viswanath Venkatesh, Michael M. G. Morris, Gordon G. B. Davis and Fred F. D. Davis. 2003. User Acceptance of Information Technology: Toward a Unified View. MIS Quarterly 27, 3, I425-478.

37. Aku Visuri, Zhanna Sarsenbayeva, Jorge Goncalves, Evangelos Karapanos and Simon Jones. 2016. Impact of Mood Changes on Application Selection. In International Joint Conference on Pervasive and Ubiquitous Computing Adjunct, ACM. http://dx.doi.org/10.1145/2968219.2968317

38. Dominik Weber, Alexandra Voit, Philipp Kratzer and Niels Henze. 2016. In-situ investigation of notifications in multi-device environments. In Proceedings of the 2016 ACM International Joint Conference on Pervasive and Ubiquitous Computing, 1259-1264.

39. Liang-Hong Wu, Liang-Chuan Wu and Shou-Chi Chang. 2016. Exploring consumers' intention to accept smartwatch. Computers in Human Behavior 64, I383-392. http://dx.doi.org/10.1016/j.chb.2016.07.005

40. Taha Yalçin. 2015. Nutzerzentrierte Untersuchung von Benachrichtigungen auf intelligenten Uhren. Grin Verlag Gmbh.

41. Tingxin Yan, David Chu, Deepak Ganesan, Aman Kansal and Jie Liu. 2012. Fast app launching for mobile 
devices using predictive user context. In MobiSys, 113-126.

http://dx.doi.org/10.1145/2307636.2307648 